\title{
Changing Role of Liver Biopsy Indications in A Tertiary Care Centre: A Histopathological Study
}

\author{
Kalaranjini KV, Jinu Abraham Glaxon* and Sheela Vasudevan
}

Department of Pathology, SreeGokulam Medical College and Research Foundation, Venjaramoodu, Kerala, India

\begin{abstract}
Background: The histomorphological study of liver biopsy is the best means to ascertain the nature and signifi $\neg$ cance of pathological processes occurring in the liver. The current non-invasive tools are unqualified to replace liver biopsy in clinical practice.

Methods: This is a one year prospective study from January 2017 to 2018. A total of twenty five cases suspected to have chronic parenchymal liver disease, inflammatory lesions or neoplastic lesions were included in the study. Detailed history and investigations done including biochemical parameters, radiological and other investigative procedures done were recorded and percutaneous liver biopsy was done using trucut needle.

Results: Out of the total 25 liver biopsy done, $60 \%$ cases were male, $40 \%$ were females. Majority of the patients belonged to the sixth decade $(32 \%)$. Youngest patient was a 12 year boy with history of intermittent jaundice since birth, and the oldest patient was a 78 year old male. The most prevalent pathological diagnosis was cirrhosis with steatohepatitis with 7 cases $(28 \%)$, followed by Non Alcoholic Fatty Liver Disease (NAFLD) and autoimmune hepatitis, $12 \%$ each.

Conclusion: Liver biopsy is the gold standard for diagnosis of chronic liver diseases and plays a valuable role in diagnosis, prognostic and management of liver diseases.
\end{abstract}

Keywords: Liver Biopsy, Chronic Liver Disease, Cirrhosis, Steatohepatitis

\section{Introduction}

Liver biopsy is the gold standard method for liver histology assessment in adults as well as among the paediatric population, mainly because of the absence of better alternatives. It gives us important information regarding parenchymal integrity, degree of fibrosis, bile duct status and deposition of materials in the liver. ${ }^{[1,2,3]}$

Liver biopsy is an important investigation and plays a valuable role in diagnosis, prognostic and management of liver diseases. The main indications are in the diagnosis and staging of parenchymal liver diseases, abnormal liver function tests of unknown aetiology, fever of unknown origin, focal or diffuse abnormalities on imaging studies and also in developing treatment plans based on histologic analysis. ${ }^{[2,3,4]}$ The different types of liver biopsies include percutaneous, transjugular, laparoscopic, fine needle aspiration and open surgery liver biopsy. ${ }^{[2,3,4]}$ However, the current non-invasive tools are unqualified to replace liver biopsy in clinical practice. Liver biopsy is the only investigation for the confirmatory diagnosis of chronic liver disease in spite of major advances in diagnostic procedures. With careful selection of patients and appropriate performance of procedure, the complications are exceptionally rare in current clinical practice.
The aim of this study is to classify liver diseases as inflammatory, non-neoplastic and neoplastic lesions, to grade and establish aetiology in cirrhosis and to determine the patterns of histological diagnosis made from liver biopsies.

\section{Materials and Methods}

This is a one year prospective study from January 2017 to January 2018. A total of twenty five cases suspected to have chronic parenchymal liver disease, inflammatory lesions or neoplastic lesions were included in the study. Detailed history, clinical features, clinical diagnosis, biochemical parameters, radiological and other investigative procedures done were recorded. After taking informed consent, percutaneous liver biopsy was done using trucut needle. Liver biopsy cores were allowed to fix in $10 \%$ formalin. All cores were submitted and haematoxylin and eosin (H\&E) stained sections were studied for histopathological examination. Hepatitis is graded by Modified Histological Activity Index (HAI) and fibrosis staging of cirrhosis was done by Ishak fibrosis staging system. Additional special stains like Masson Trichrome, reticulin and Periodic Acid Schiff (PAS), were used to highlight and identify features that were not easily seen on $\mathrm{H} \& \mathrm{E}$ stain. The choice of special stains depends on the findings on the initial assessment, clinical details and the preference of the pathologist. 


\section{Results}

Out of 25 patients, $15(60 \%)$ were males and $10(40 \%)$ females, with a male to female ratio of $1.5: 1$. Table 1 shows the age distribution of the patients. Majority of the patients belonged to the sixth decade (32\%). Youngest patient was a 12 year boy with history of intermittent jaundice since birth, and the oldest patient was a 78 year old male.

Table 2 shows the various clinical presentations of the cases. Most of the patients, 15 cases $(60 \%)$ presented with dull aching abdominal pain in right hypochondrium and jaundice.

The distribution of various pathological conditions is given in Table 3. Among the 25 cases, the most prevalent was cirrhosis with steatohepatitis with 7 cases $(28 \%)$, followed by NAFLD and autoimmune hepatitis, $12 \%$ each.
In all relevant cases, the HAI score and Ishak fibrosis staging score was done (Table 4) Among the seven cases of cirrhosis with steatohepatitis, majority (6 cases) showed Ishak fibrosis score of 6 and one case with fibrosis score 5.

In all cases, relevant liver function tests were done. Serum bilirubin was found to be elevated $(>1 \mathrm{gm} / \mathrm{dl})$ in 7 cases $(28 \%)$. None of the patients were HIV, HBV or HCV positive.

\section{Discussion}

The world health statistics 2007-2009 indicate that chronic liver disease occurs in age group of more than 55 years with steady increase in death rate up to $75+$ years. ${ }^{[1,5]}$ In this study, majority of the individuals suffering from chronic liver disease were in 40-60 years of age with male preponderance. Similar results of male predominance is

Table 1: Age distribution of patients.

\begin{tabular}{|c|c|c|}
\hline Age distribution(years) & No of cases (\%) & Percentage (\%) \\
\hline $11-20$ & 1 & $16 \%$ \\
\hline $31-40$ & 4 & $24 \%$ \\
\hline $41-50$ & 6 & $16 \%$ \\
\hline $51-60$ & 4 & $32 \%$ \\
\hline $61-70$ & 8 & $08 \%$ \\
\hline $71-80$ & 2 & $100 \%$ \\
\hline TOTAL & $\mathbf{2 5}$ & 2 \\
\hline
\end{tabular}

Table 2: Clinical presentation of the cases.

\begin{tabular}{|c|c|c|}
\hline Clinical presentation & Number of cases & Percentage (\%) \\
\hline Abdominal pain & 8 & $28 \%$ \\
\hline Jaundice & 7 & $16 \%$ \\
\hline Weight loss & 4 & $16 \%$ \\
\hline Ascites & 4 & $08 \%$ \\
\hline Fever & 2 & $\mathbf{1 0 0 \%}$ \\
\hline TOTAL & $\mathbf{2 5}$ & \\
\hline
\end{tabular}

Table 3: Histopathological distribution of cases.

\begin{tabular}{|c|c|c|}
\hline Histopathological Diagnosis & No. of Cases & Percentage(\%) \\
\hline Cirrhosis with Steatohepatitis & 7 & $28 \%$ \\
\hline Non Alcoholic Fatty Liver Disease & 3 & $12 \%$ \\
\hline Autoimmune hepatitis & 3 & $12 \%$ \\
\hline Steatosis & 2 & $08 \%$ \\
\hline Metastasis to liver & 2 & $08 \%$ \\
\hline Steatohepatitis & 1 & $04 \%$ \\
\hline Chronic hepatitis & 1 & $04 \%$ \\
\hline Chronic hepatitis with cirrhosis and autoimmune hepatitis & 1 & $04 \%$ \\
\hline Chronic liver disease & 1 & $04 \%$ \\
\hline
\end{tabular}




\begin{tabular}{|c|c|c|}
\hline Histopathological Diagnosis & No. of Cases & Percentage(\%) \\
\hline Methotrexate toxicity & 1 & $04 \%$ \\
\hline Benign biliary cyst & 1 & $04 \%$ \\
\hline Cirrhosis with hepatocellular Carcinoma & 1 & $04 \%$ \\
\hline BRIC 2 & 1 & $04 \%$ \\
\hline GRAND TOTAL CASES & 25 & $100 \%$ \\
\hline
\end{tabular}

Table 4: HAI score and Ishak fibrosis staging score.

\begin{tabular}{|c|c|c|c|}
\hline Histopathological Diagnosis & Cases (\%) & $\mathrm{HAl}$ & Fibrosis \\
\hline Cirrhosis with Steatohepatitis & $\begin{array}{l}\text { Case } 1 \\
\text { Case } 2 \\
\text { Case } 3 \\
\text { Case } 4 \\
\text { Case } 5 \\
\text { Case } 6 \\
\text { Case } 7\end{array}$ & $\begin{array}{c}7 / 18 \\
3 / 18 \\
2 / 18 \\
11 / 18 \\
11 / 18 \\
- \\
-\end{array}$ & $\begin{array}{l}6 \\
6 \\
6 \\
6 \\
6 \\
6 \\
5\end{array}$ \\
\hline Autoimmune hepatitis & $\begin{array}{l}\text { Case } 1 \\
\text { Case } 2 \\
\text { Case } 3\end{array}$ & $\begin{array}{l}5 / 18 \\
5 / 18 \\
5 / 18 \\
\end{array}$ & $\begin{array}{l}3 \\
4 \\
3\end{array}$ \\
\hline Steatosis & $2(08 \%)$ & - & - \\
\hline Steatohepatitis & $1(04 \%)$ & $3 / 18$ & 4 \\
\hline Chronic hepatitis & $1(04 \%)$ & - & - \\
\hline Chronic hepatitis with cirrhosis and autoimmune hepatitis & $1(04 \%)$ & $6 / 18$ & 6 \\
\hline Chronic liver disease & $1(04 \%)$ & $6 / 18$ & 1 \\
\hline Cirrhosis with hepatocellular Carcinoma & $1(04 \%)$ & $6 / 18$ & 6 \\
\hline
\end{tabular}

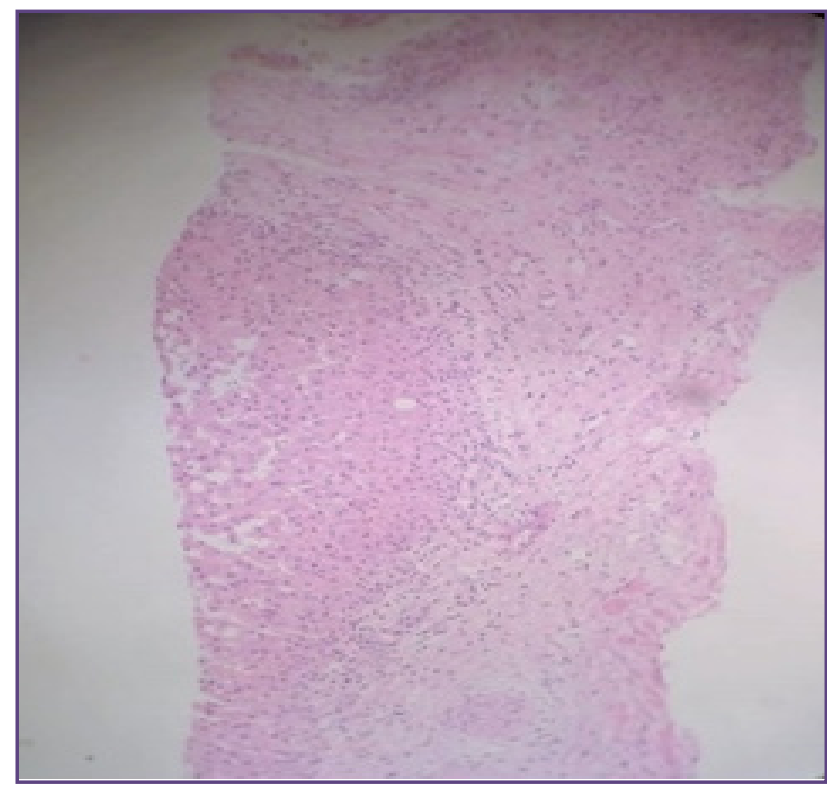

Fig. 1: Liver biopsy showing nodules separated by fibrous septae in cirrhosis. (H\&E 10x).

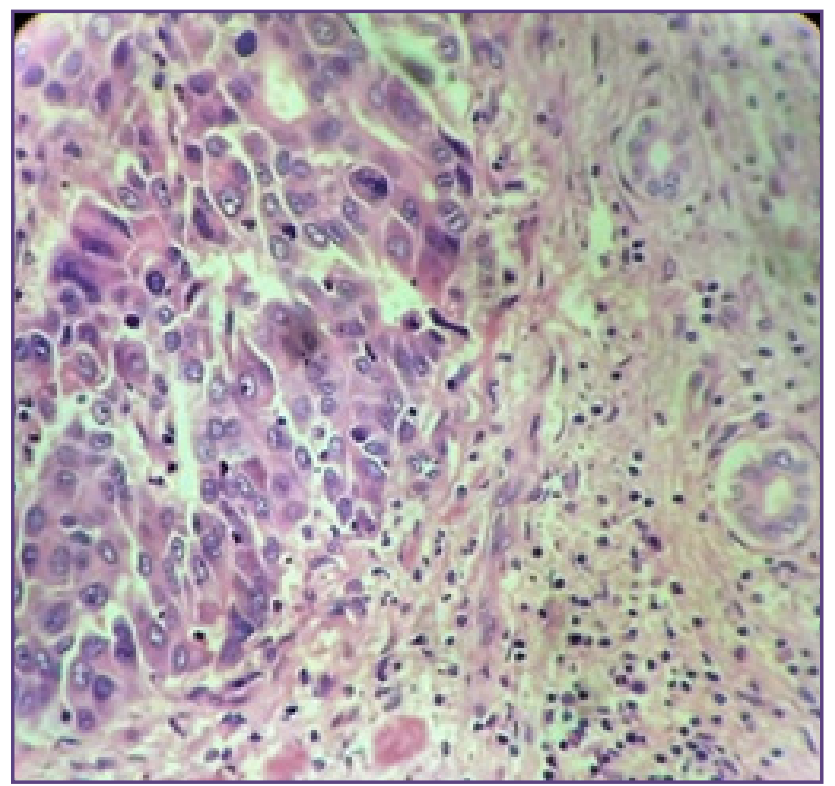

Fig. 2: Liver biopsy in a case of HCC with cirrhosis (H\&E 10x). 


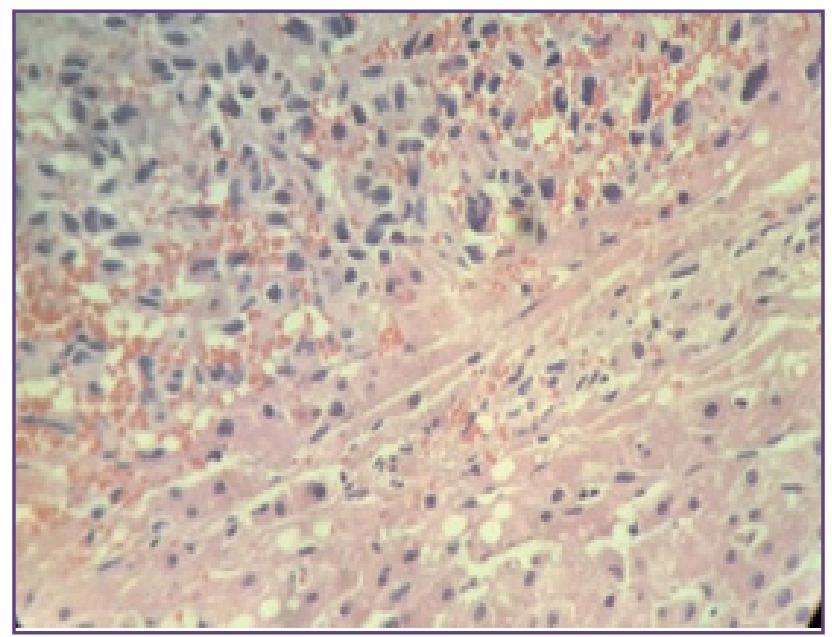

Fig. 3: Liver biopsy showing hepatic metastasis with primary tumor being in adrenal (malignant pheochromocytoma).

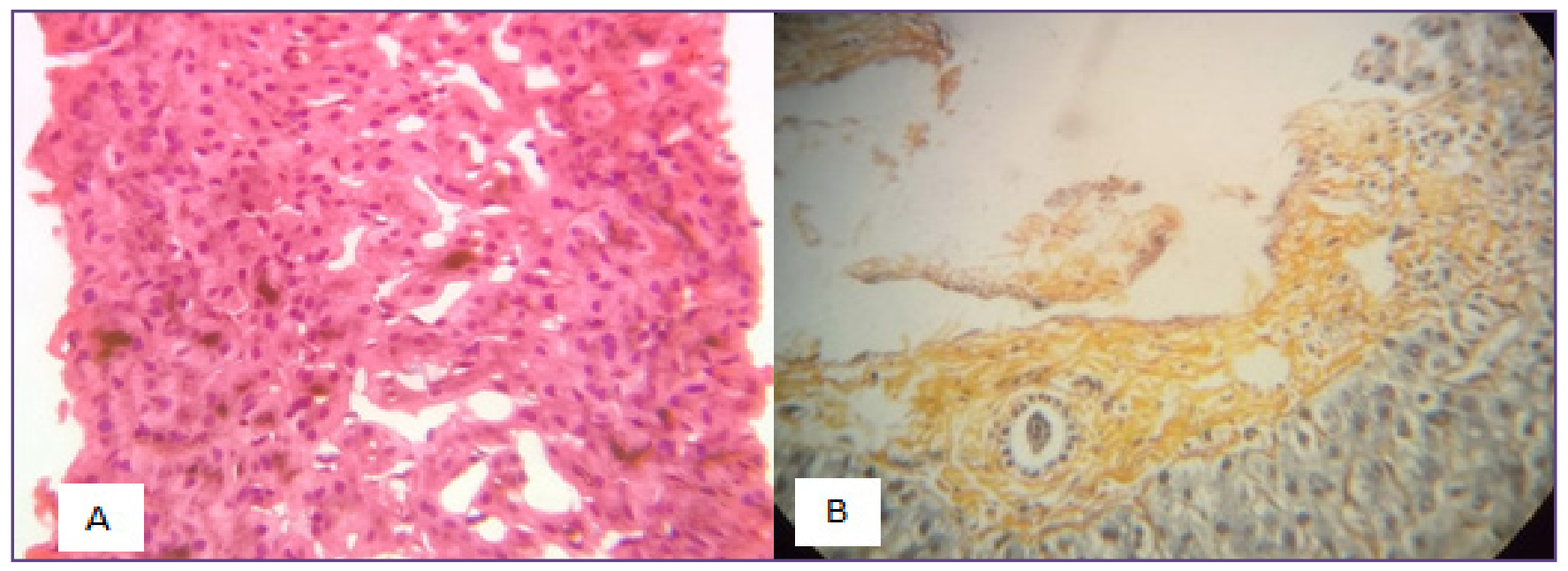

Fig: 4A. Liver biopsy showing marked intrahepatic and canalicular cholestasis in a case of BRIC (H\&E 10x) B. Special stain demonstrating periportal fibrosis (Reticulin 10x).

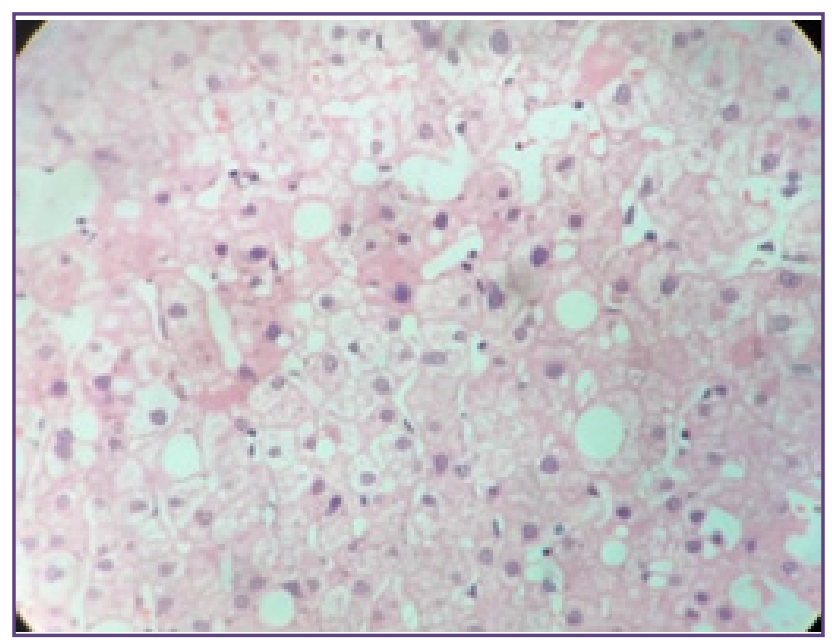

Fig. 5: Liver biopsy in methotrexate induced liver injury showing macrovesicular steatosis and moderate anisonucleosis(H\&E 10x). 
reported in other studies..$^{[1,5,6,7]}$ The cause for this is most probably due to alcohol intake which is highest among middle aged males all over the world. However, in a study by Drebber et al. ${ }^{[8]}$, it was found that females were affected more than males.

In the present study the common presenting symptoms were abdominal pain (32\%), jaundice (28\%), weight loss $(16 \%)$, ascites $(16 \%)$ and fever $(08 \%)$, while $16 \%$ cases had hepatomegaly clinically. Liver biopsy done in these patients yielded a diagnosis in all cases. These findings were consistent with similar studies done by Chawla et al ${ }^{[1]}$ where $74 \%$ of cases complained of abdominal pain with discomfort while $42 \%$ had hepatomegaly clinically. In another series ${ }^{[9]}, 10 \%$ patients had abdominal pain while $48 \%$ patients had hepatomegaly.

Liver cirrhosis with steatohepatitis (Figure 1) was the most common lesion $(28 \%)$ in this study. This is most probably attributable to increase alcohol intake as well as the fact that it is the end stage of many hepatic diseases. This incidence correlates with the findings of Mc Sween ${ }^{[10]}$ who reviwed the incidence of cirrhosis in different geographical areas and found the rate of cirrhosis in necropsy to be between $0.6-29.1 \%$.

In this study, Non Alcoholic Steatohepatitis (NASH) was the second most common lesion with 3 cases $(12 \%)$. The histopathological diagnosis of NASH included steatosis with ballooning degeneration was seen from zone 1 to zone 3 with lobular inflammation comprising of predominantly neutrophils or lymphocytes in different patients. Perisinusoidal fibrosis was noted. Biopsy performed by Verma et al ${ }^{[1]}$ found $27 \%$ cases had NASH diagnosed on liver biopsy while Laurin ${ }^{[8]}$ found $2-3 \%$ cases of NASH on liver biopsy after excluding viral, metabolic, autoimmune, and genetic causes of chronic liver disease.

Three cases $(12 \%)$ of autoimmune hepatitis was seen in this study. Histopathological findings showed ballooning degeneration with cirrhosis, lobular and periportal inflammation and portal to portal bridging fibrosis.

In the present study, steatosis and steatohepatitis was the third most common lesion seen in $12 \%$ cases. The usual causes are alcohol intake, obesity, diabetes mellitus, consumption of drugs like estrogen, steroids and surgical procedures like jejunoileal bypass and small bowel resection. Similar findings were observed by Chawla et al ${ }^{[8]}$ where steatosis seen in $20 \%$ cases.

In the present study one case of $\mathrm{HCC}$ with cirrhosis (Figure 2) was observed. Metastatic involvement of liver is more common than HCC. Most common primaries producing hepatic metastasis are breast, lung and colon respectively. In the present study two cases of metastatic adenocarcinomas were observed with primary tumor being in adrenal (malignant pheochromocytoma) and pancreas respectively (Figure 3). Similar findings were observed in series of studies..$^{[1,5,8]}$

Liver biopsy done in a 12 year old boy with history of intermittent jaundice since birth, now presenting with fever, itching and jaundice. Histopathology showed liver biopsy with marked intrahepatic and canalicular cholestasis. Gamma glutamyl transferase studies was found to be reduced. Immunohistochemistry (IHC) for canalicular molecular markers MDR3 and BSEP was positive. DNA studies done showed homozygous missense variation in exon 26 of ABCB11 gene. Correlating with the diagnostic criteria for Benign Recurrent Intrahepatic Cholestasis (BRIC) by Luketic and Shiffman ${ }^{[11]}$, the IHC results and the DNA studies, the diagnosis of BRIC 2 (Figure 4) was given. BRIC is a rare autosomal recessive disorder characterised by intermittent episodes of cholestasis with a benign course without progression to liver failure. Genetic studies have located the defective gene ATP8B1 on the long arm of chromosome 18 . The defective gene encodes the FIC1 protein which is associated with PFIC type 1. Another genetically distinct form of BRIC is associated with mutations in ABCB11 which encodes the human export bile salt export pump. Approximately 100 cases of BRIC have been reported till date. ${ }^{[11,12,13]}$

In a case of a 47 year old female with psoriasis with history of methotrexate intake with a high cumulative dose since 2 years. Liver biopsy was done which showed hepatocytes with mild ballooning degeneration, mild macrovesicular steatosis and mild periportal fibrosis. The hepatocytes showed moderate anisonucleosis and mild lobular inflammation (Figure 5). The diagnosis consistent with liver injury by methotrexate Grade 3A as per Roengik system. The Roengik classification was developed by Psoriasis task force led by dermatologists and is based on clinical observations; it has since been recommended by American Academy of Dermatology guidelines for monitoring methotrexate induced liver injury. ${ }^{[14,15]}$

\section{Conclusion}

Liver biopsy study is the gold standard for diagnosis of chronic liver diseases. Microscopic examination of liver biopsy helps to classify liver diseases as inflammatory, nonneoplastic and neoplastic lesions, to grade and establish aetiology in cirrhosis and to determine the patterns of histological diagnosis thereby providing better treatment options. 


\section{References}

1. Verma A, Sinha S, Panicker NK. The clinicopathological correlation in suspected cases of chronic liver disease with the aid of liver biopsy - a study in tertiary health centre. Int J Med Sci Public Health 2016;5:2615-19.

2. Torbenson M. Biopsy Interpretation of the liver. 3rd ed. Philadelphia PA: Wolter Kluwer; 2015.

3. Burt A, Portmann B, Ferrell L. MacSween's pathology of the liver. 6th ed. London: Churchill Livingstone; 2012.

4. Robert D. Odze, John R. Goldblum. Odze And Goldblum Surgical Pathology of the GI Tract, Liver, Biliary Tract, and Pancreas. Philadelphia, PA :Saunders/Elsevier; 2015.

5. Gao ZH, Tretiakova MS, Liu WH, Gong C, Farris PD, Hart J. Association of E-cadherin, matrix metalloproteinases, and tissue inhibitors of metalloproteinases with the progression and metastasis of hepatocellular carcinoma. Mod Pathol 2006; 19: 533-40.

6. Wang CH, Mo LR, Chang KK, Lin RC, Kuo JJ. A cohort study to investigate hepatocellular carcinoma risk in hepatitis C patients. Hepatogastroenterology. 2011 MayJun; 58: 904-8.

7. Gelb AM, Mildvan D, Stenger RJ. The spectrum and causes of liver diseases in narcotic addicts. Am J Gastroenterol. 1977 Apr; 67: 314-8.

8. Chawla N, Sunila S. Spectrum of Histopathological Findings in Liver Biopsy. Indian Medical Gazette. 2013 Oct 363-7.

9. Laurin J. Motion - all patients with NASH need to have a liver biopsy: arguments against the motion. Can J Gastroenterol. 2002 Oct; 16: 722-6.

10. MacSween R.N.M., Scott A.R. - Hepatic cirrhosis: A Clinicopathological review of 520 cases. J ClinPathol. 26:936- 42, 1973.

11. Luketic VA, Schiffman ML. Benign recurrent intrahepatic cholestasis. Clin Liver Dis 1999;3:509-28.

12. Geethalakshmi S, Mageshkumar S. Benign Recurrent Intrahepatic Cholestasis: A Rare Case Report. Int J Sci Stud 2014;2(7):222- 4.

13. Gupta V, Kumar M, Bhatia BD. Benign recurrent intrahepatic cholestasis. Indian J Pediatr 2005;72:793-4

14. Ferrell L, Kakkar S. Liver pathology. 4th ed. New York: Demos Medical Publishing; 2012.

15. Aithal GP, Haugk B, Das S et al. Monitoring methotrexateinduced hepatic fibrosis in patients with psoriasis: are serial liver biopsies justified? Aliment Pharmacol Ther; 2004 Feb $15 ; 19(4): 391-9$.

*Corresponding author:

Dr. Jinu Abraham Glaxon, Associate Professor, Department Of Pathology, SreeGokulam Medical College and Research Foundation, Venjaramoodu, Kerala - 695607 INDIA

Phone: +919544800523

Email: jinu232@gmail.com

Financial or other Competing Interests: None. 